\title{
ATUAÇÃO DO ENFERMEIRO DO TRABALHO NA SAÚDE E PROTEÇÃO DOS TRABALHADORES OFFSHORE
}

\section{Work of nursing practice in health and protection of offshore workers}

Claudemir Santos de Jesus ${ }^{1}$, Fabiano de Oliveira², Mônica Candido da Rocha ${ }^{3}$, Rogéria Maria Silva do Nascimento ${ }^{4}$

${ }^{1}$ Enfermeiro, Mestre em Enfermagem pela EEAN/UFRJ, Professor Convidado pela Universidade Iguaçu.

${ }^{2}$ Enfermeiro, Especialista em Enfermagem do trabalho pela Universidade Iguaçu.

${ }^{3}$ Enfermeira, Especialista em Enfermagem do trabalho pela Universidade Iguaçu.

${ }^{4}$ Enfermeira, Mestre em Enfermagem pela EEAN/UFRJ, Coordenadora do Curso de Enfermagem do trabalho pela Universidade Iguaçu.

\section{Endereço para correspondência:}

Claudemir Santos de Jesus

Rua São Francisco Xavier, 859, Apt. 1002, BI. A, Maracanã, Rio de Janeiro-RJ.

Telefone: (21) 96487-0169.

E-mail: udemi34@yahoo.com.br 


\title{
Resumo
}

Trata-se de um estudo que objetivou identificar a atuação do enfermeiro nas ações da promoção, prevenção de doenças ocupacionais e manutenção da saúde dos trabalhadores embarcados. Utilizou-se abordagem qualitativa, baseada em revisão integrativa - cuja questão de pesquisa foi: Quais as produções científicas que retratam a atuação do enfermeiro nas ações da promoção, prevenção de doenças ocupacionais e manutenção da saúde dos trabalhadores embarcados? - através da biblioteca virtual da saúde, no período de fevereiro a outubro de 2015. Na discussão, evidenciou-se a importância do enfermeiro do trabalho no ambiente offshore, atuando na prevenção de doenças e acidentes de trabalho, e desenvolvendo um papel constante de promoção e proteção da saúde do trabalhador. Concluimos que o serviço do enfermeiro do trabalho no ambiente offshore é envolvido com a promoção da saúde, prevenção de agravos e acidentes ocupacionais, mas percebemos também no estudo a escassez de produções relacionadas à temática em questão, nos bancos de dados virtuais.

Palavras-chave: Qualidade de vida; Enfermagem; Saúde do trabalhador.

\begin{abstract}
It is a study aimed at identifying the role of the nurse in the actions of promotion, prevention of occupational diseases and health maintenance of embedded workers. We used a qualitative approach, based on integrative review, whose research question was: What are the scientific productions that portray the work of nurses in the actions of promotion, prevention of occupational diseases and maintaining the health of workers on board? Through the Virtual Library of Health, from February to October 2015. In the discussion, it highlighted the importance of the work of nurses in the offshore environment acting on the prevention of illness and accidents at work, but developing a steady role in promoting and protecting health worker. We conclude that the work of the nursing service in the offshore environment is involved in health promotion, disease prevention and occupational accidents, but also realize the study the shortage of productions related to themes concerned in virtual databases.
\end{abstract}

Keywords: Quality of life; Nursing; Occupational Health. 
Com base na importância de refletir sobre as atividades do enfermeiro num contexto offshore, o estudo visa mostrar as ações, perfil e as relações deste profissional com os trabalhadores embarcados ${ }^{1}$. O estudo objetivou identificar a atuação do enfermeiro nas ações da promoção, prevenção de doenças ocupacionais e manutenção da saúde dos trabalhadores embarcados.

Martinho² descreve que "ao longo da história, o trabalho sempre ocupou um espaço central no mundo dos homens. Entendido como práxis...". Assim, é comum observar nos trabalhadores embarcados o sentimento do "homem" que não adoece e nem precisa de ajuda, sendo um desafio à atuação do enfermeiro do trabalho, que tem $\mathrm{o}$ intuito de produzir ações eficientes e eficazes perante as orientações $e$ determinações legais brasileiras, fundamentais na promoção da saúde e na manutenção da integridade física e psíquica dos trabalhadores embarcados ${ }^{3}$.

Dessa forma, o enfermeiro possibilita informar e instruir a classe trabalhadora embarcada sobre a importância da qualidade de vida, através da reflexão sobre a promoção do auto-cuidado desses indivíduos, para assim diminuir a barreira cultural machista em relação ao adoecer ${ }^{4}$.

Frente às questões trabalhistas, como a Consolidação das Leis do Trabalho, a Constituição Federal de 1988 garante a saúde do trabalhador como direito social e competência do Sistema Único de Saúde (SUS), e as normas regulamentadoras avaliam a segurança do trabalhador ${ }^{5}$.

O enfermeiro offshore articula conhecimentos e gerencia práticas que consistem na prestação das ações desenvolvidas aos trabalhadores a bordo nas plataformas, com foco na manutenção e promoção da saúde ocupacional, apoio psicossocial, prevenções de doenças e riscos, com estímulo do uso adequado dos equipamentos de proteção individual para evitar situações de emergência e urgência no trabalho'.

É primordial a qualificação das práticas à atuação com os profissionais embarcados, principalmente pela construção do processo de conhecimentos, perfazendo uma busca de qualidade de vida pessoal e intelectual do trabalhador, com base no marco constitucional que designa o SUS ${ }^{6}$. 
As plataformas de petróleo requerem o enfermeiro com perfil profissional diferenciado, de competências para o desenvolvimento do processo de trabalho nos espaços de atuação, que estabeleça junto a equipe multiprofissional uma interação, dialoga e implementa ações ao trabalhador que exerce atividades perigosas, em um espaço dito confinado, baseado numa escala de 14 dias de trabalho contínuo embarcado e 14 dias de folga ${ }^{7}$.

Em resumo, o enfermeiro offshore tem como atuação a aplicação dos primeiros socorros, ações de inspeção, fiscalização, análise da qualidade da água, segurança, orientação dos hábitos alimentares, higienização, atividades físicas, descanso e atestado de saúde ocupacional, independente da área de atuação dos trabalhadores, que nesse ambiente de trabalho se faz de forma companheira e solidária pelo distanciamento social, provocando uma quebra da rotina familiar e com os amigos que não trabalham embarcados ${ }^{8}$.

Um fato relevante das dificuldades de trabalho do enfermeiro é o ambiente com fatores constantes e simultâneos que interagem no processo de labuta do trabalhador, como vibrações, ruídos, baixa luminosidade e ventilação inadequada, o que dificulta a adaptação física, biológica e psicológica, podendo gerar estresse e transtornos de natureza psicossocial ${ }^{9}$.

Baseado nos princípios da profissão de enfermagem, a exigência para o enfermeiro é ter habilidade para desenvolver suas atribuições com base no valor humano, para um grau de satisfação que implique no respeito pelos funcionários para auxiliar na qualidade e produtividade, num cenário em que é o único profissional da saúde com turno diário de 12 horas de trabalho e 12 de descanso, com sobreaviso para atendimento de emergências.

Vilela e Souza ${ }^{10}$ reforçam que a liderança "envolve compromisso, responsabilidade, empatia, habilidade para tomada de decisões, comunicação e gerenciamento de forma efetiva e eficaz". O enfermeiro offshore visa a garantia da assistência e a segurança do trabalhador e também nas situações de acidente as quais haja necessidade de resgate aeromédico, procedendo ao transporte de emergência a uma unidade hospitalar com recursos assistências mais complexos. 
Analisando esse contexto e confrontando com a realidade da saúde dos trabalhadores embarcados, todas estas questões são voltadas para a existência de funcionários mais saudáveis e conscientes sobre o interesse do cuidar.

$O$ estudo tem $o$ intuito de contribuir para o ensino, assistência, pesquisa e extensão do assunto abordado, no intuito de facilitar e colaborar com a pesquisa nessa área, tendo uma abordagem pertinente a temática dos trabalhadores embarcados.

\section{MÉTODOS E RESULTADOS}

Trata-se de estudo descritivo, que investigou as situações de atuação do enfermeiro do trabalho para o trabalhador embarcado. A abordagem qualitativa da pesquisa tem por meta o entendimento intrínseco de uma dada realidade, onde seu foco encontra-se no particular e não no genérico ${ }^{11}$.

A pesquisa bibliográfica propicia o exame de um tema sob nova abordagem, sendo utilizada para o desenvolvimento de uma temática sob a análise de publicações, reconhecendo-se sua regularidade, tipos, assuntos examinados por intermédio de artigos científicos, tese, monografias.

Baseado nas seis etapas da revisão integrativa, na qual utilizamos a biblioteca virtual em saúde, no período de fevereiro a outubro de 2015, em sua primeira etapa elaboramos a questão de pesquisa, que foi: Quais as produções científicas que retratam a atuação do enfermeiro nas ações da promoção, prevenção de doenças ocupacionais e manutenção da saúde dos trabalhadores embarcados?

Na segunda fase fizemos uma intensa busca das produções, definindo, assim, os descritores consultados no DeCS - Terminologia em Saúde: Qualidade de Vida; Enfermagem; Saúde do Trabalhador; Plataforma. Foi realizada uma busca refinada com descritores isolados e associados para responder ao objetivo da pesquisa.

Para tal, definimos os critérios de inclusão, que foram: artigos e teses na íntegra, em português, que retratassem a temática, no período de 2010 a 2015. Isto permitiu a seleção das publicações, análise dos conteúdos, classificação e categorização das características do ambiente de trabalho da plataforma offshore nas bases de dadosLiteratura Latino-Americana e do Caribe em ciências da Saúde (LILACS), Base de dados de enfermagem (BDENF), Medical Literature Analysis and Retrieval System Online (MEDLINE). 
Ressaltamos que os critérios de exclusão estabelecidos foram: indisponibilidade de acesso, publicações duplas, resumo, textos na forma de projetos, artigos/trabalhos incompletos e artigos/trabalhos em outros idiomas, fora do recorte temporal e os que não são articulados à temática. O quadro 1 apresenta os resultados da busca realizada com os descritores isoladamente e associados, de todas as produções relacionadas ou não com a temática.

Quadro 01. Resultados da busca com descritores.

\begin{tabular}{|c|c|c|c|c|}
\hline \multirow{2}{*}{ DESCRITORES ISOLADOS } & \multicolumn{4}{|c|}{ BASES DE DADOS } \\
\hline & LILACS & BDENF & MEDLINE & TOTAL \\
\hline Enfermagem & 6209 & 3677 & 934 & 10.820 \\
\hline Qualidade de vida & 3161 & 421 & 361 & 3.943 \\
\hline Saúde do trabalhador & 1777 & 400 & 250 & 2.427 \\
\hline Plataforma & 264 & 08 & 02 & 274 \\
\hline Total & 11411 & 4506 & 1547 & 17.464 \\
\hline \multirow{2}{*}{ DESCRITORES ASSOCIADOS } & \multicolumn{4}{|c|}{ BASES DE DADOS } \\
\hline & LILACS & BDENF & MEDLINE & TOTAL \\
\hline $\begin{array}{l}\text { Enfermagem and Qualidade de vida } \\
\text { and Saúde do trabalhador and } \\
\text { Plataforma }\end{array}$ & 0 & 0 & 0 & 0 \\
\hline $\begin{array}{l}\text { Enfermagem and Qualidade de vida } \\
\text { and Saúde do trabalhador }\end{array}$ & 37 & 28 & 01 & 66 \\
\hline Enfermagem and Qualidade de vida & 373 & 249 & 17 & 639 \\
\hline $\begin{array}{l}\text { Enfermagem and Qualidade de vida } \\
\text { and Plataforma }\end{array}$ & 01 & 0 & 0 & 01 \\
\hline Enfermagem and Plataforma & 19 & 07 & 02 & 28 \\
\hline Total & 430 & 284 & 20 & 734 \\
\hline
\end{tabular}

Fonte: Próprio Autor

A busca através da Biblioteca Virtual em Saúde, seguindo os critérios de inclusão, foi extremamente exaustiva, por causa do quantitativo encontrado de 17.464 estudos na plataforma na primeira filtragem. Mas ao realizar a associação dos descritores o quantitativo declinou para 734 produções, sendo assim, foram excluídos do estudo 16.730 produtos.

A partir desses, numa segunda filtragem, pudemos examinar os achados, para assim obter 07 publicações, que entraram na terceira fase. Nessa fase utilizamos um instrumento que teve como objetivo extrair os resultados pelas informações 


\section{Revisão}

\section{Saúde Funcional}

chaves de cada produção selecionada na busca dos descritores relacionados à temática, na qual identificamos as bases de dados, como podemos visualizar no quadro 2 abaixo.

Quadro 02. Resultados da busca com descritores relacionados à temática.

\begin{tabular}{|l|c|c|c|c|}
\hline \multicolumn{1}{|c|}{ DESCRITORES ASSOCIADOS } & \multicolumn{4}{c|}{ BASES DE DADOS } \\
\cline { 2 - 5 } & LILACS & BDENF & MEDLINE & TOTAL \\
\hline $\begin{array}{l}\text { Enfermagem and Qualidade de vida and } \\
\text { Saúde do trabalhador and Plataforma }\end{array}$ & 0 & 0 & 0 & 0 \\
\hline $\begin{array}{l}\text { Enfermagem and Qualidade de vida and } \\
\text { Saúde do trabalhador }\end{array}$ & 01 & 0 & 01 & 02 \\
\hline Enfermagem and Qualidade de vida & 02 & 01 & 0 & 03 \\
\hline $\begin{array}{l}\text { Enfermagem and Qualidade de vida and } \\
\text { Plataforma }\end{array}$ & 01 & 0 & 0 & 01 \\
\hline Enfermagem and Plataforma & 0 & 0 & 01 & 01 \\
\hline \multicolumn{1}{|c|}{ Total } & 04 & 01 & 02 & 07 \\
\hline
\end{tabular}

Fonte: Próprio Autor

Ao cumprir a quarta fase, as produções selecionadas para revisão integrativa foram analisadas para a verificação de sua autenticidade, qualidade metodológica, importância das informações e representatividade. Para tal, construímos um quadro em que destacamos o periódico, base de dados, ano, local, título e metodologia e o tipo de formato da literatura, como visto a seguir:

Quadro 03. Produções Coletadas da busca dos descritores relacionados à temática.

\begin{tabular}{|c|c|c|c|c|c|c|}
\hline Periódico & Base & Ano & Local & Título & Metodologia & Tipo \\
\hline $\begin{array}{c}\text { Revista } \\
\text { UERJ }\end{array}$ & LILACS & 2011 & RJ & $\begin{array}{c}\text { Cacterísticas do } \\
\text { ambiente de } \\
\text { trabalho do } \\
\text { enfermeiro em } \\
\text { plataforma de } \\
\text { petróleo offshore }\end{array}$ & $\begin{array}{c}\text { Revisão } \\
\text { bibliográfica }\end{array}$ & $\begin{array}{c}\text { Artigo de } \\
\text { Revisão }\end{array}$ \\
\hline UFF & LILACS & 2012 & RJ & $\begin{array}{c}\text { Trabalho e } \\
\text { transtornos } \\
\text { mentais: uma } \\
\text { análise da saúde } \\
\text { mental dos } \\
\text { petroleiros da } \\
\text { Bacia de Campos } \\
\text { frente as }\end{array}$ & $\begin{array}{c}\text { Pensamento } \\
\text { histórico, } \\
\text { crítico e } \\
\text { dialético, } \\
\text { Pesquisa de } \\
\text { Campo }\end{array}$ & $\begin{array}{c}\text { Trabalho de } \\
\text { Conclusão } \\
\text { de Curso }\end{array}$ \\
\hline
\end{tabular}




\begin{tabular}{|c|c|c|c|c|c|c|}
\hline & & & & $\begin{array}{l}\text { transformações no } \\
\text { mundo do trabalho }\end{array}$ & & \\
\hline $\begin{array}{c}\text { Acta } \\
\text { paul. } \\
\text { enferm. }\end{array}$ & BDENF & 2012 & SP & $\begin{array}{c}\text { Trabalhador } \\
\text { portuário: perfil de } \\
\text { doenças } \\
\text { ocupacionais } \\
\text { diagnosticadas em } \\
\text { serviço de saúde } \\
\text { ocupacional }\end{array}$ & $\begin{array}{l}\text { Estudo } \\
\text { quantitativo } \\
\text { descritivo } \\
\text { com análise } \\
\text { retrospectiva }\end{array}$ & $\begin{array}{l}\text { Artigo } \\
\text { Original }\end{array}$ \\
\hline $\begin{array}{l}\text { Revista } \\
\text { UERJ }\end{array}$ & LILACS & 2012 & RJ & $\begin{array}{l}\text { Discutindo e } \\
\text { refletindo sobre a } \\
\text { competência do } \\
\text { enfermeiro } \\
\text { offshore }\end{array}$ & $\begin{array}{c}\text { Estudo } \\
\text { exploratório e } \\
\text { qualitativo }\end{array}$ & $\begin{array}{l}\text { Artigo } \\
\text { Original }\end{array}$ \\
\hline $\begin{array}{l}\text { Revista } \\
\text { Acred }\end{array}$ & MEDLINE & 2012 & RJ & $\begin{array}{l}\text { Trabalho do } \\
\text { enfermeiro } \\
\text { offshore e a } \\
\text { interface da } \\
\text { cultura de } \\
\text { segurança }\end{array}$ & $\begin{array}{l}\text { Relato de } \\
\text { experiência }\end{array}$ & $\begin{array}{c}\text { Relato de } \\
\text { experiência }\end{array}$ \\
\hline UNIRIO & MEDLINE & 2012 & RJ & $\begin{array}{l}\text { As competências } \\
\text { do enfermeiro no } \\
\text { processo de } \\
\text { trabalho em } \\
\text { plataforma } \\
\text { offshore de } \\
\text { petróleo }\end{array}$ & $\begin{array}{c}\text { Exploratório } \\
\text { com } \\
\text { abordagem } \\
\text { qualitativa }\end{array}$ & $\begin{array}{r}\text { Dissertação } \\
\text { de Mestrado }\end{array}$ \\
\hline $\begin{array}{c}\text { Texto } \\
\text { contexto - } \\
\text { enferm. }\end{array}$ & LILACS & 2013 & SC & $\begin{array}{l}\text { Enfermeiro } \\
\text { embarcado em } \\
\text { plataforma } \\
\text { petrolífera: um } \\
\text { relato de } \\
\text { experiência } \\
\text { offshore }\end{array}$ & $\begin{array}{l}\text { Relato de } \\
\text { experiência }\end{array}$ & $\begin{array}{c}\text { Relato de } \\
\text { experiência }\end{array}$ \\
\hline
\end{tabular}

Fonte: Próprio Autor

No quadro 3 acima identificam-se os seguintes periódicos e número de publicações: Revista UERJ (02); UFF (01); Acta paul. enferm. (01); Revista Acred (01); UNIRIO (01); Texto contexto - enferm. (01) e as bases de dados LILACS (04); MEDLINE (02) e BDENF (01).

Já quanto ao ano de publicação, 2012 (05) foi o ano que mais publicou sobre a temática, seguido de 2013 (01) e 2011 (01). Porém, quanto ao local de publicação dos estudos predominou o Rio de Janeiro (05), observando-se que as revistas 


\section{Revisão}

\section{Saúde Funcional}

cariocas deram mais importância; este foi seguido por São Paulo (01) e Santa Catarina (01).

Em relação à metodologia, verificamos: Relato de experiência (02); Revisão bibliográfica (01); Pensamento histórico, crítico e dialético, Pesquisa de Campo (01); Estudo quantitativo descritivo com análise retrospectiva (01); Estudo exploratório e qualitativo (01); Exploratório com abordagem qualitativa (01).

No tipo de produção foram vistos: Artigo Original (02); Relato de experiência (02); Artigo de Revisão (01); Dissertação de Mestrado (01); Trabalho de Conclusão de Curso (01).

Na quinta fase da revisão, interpretamos as informações das produções, que resultou no surgimento da seguinte categoria: a atuação do enfermeiro nas ações da promoção, prevenção de doenças ocupacionais e manutenção da saúde dos trabalhadores embarcados. Segue a baixo o quadro 4 da categoria para a discussão.

Quadro 04. Categorização das Temáticas do Estudo.

\begin{tabular}{|c|c|}
\hline \multicolumn{2}{|c|}{ TEMÁTICAS DO ESTUDO } \\
\hline CATEGORIA & TÍTULO \\
\hline \multirow{7}{*}{$\begin{array}{l}\text { Atuação do enfermeiro nas } \\
\text { ações da promoção, } \\
\text { prevenção de doenças } \\
\text { ocupacionais e manutenção da } \\
\text { saúde dos trabalhadores } \\
\text { embarcados }\end{array}$} & $\begin{array}{l}\text { Características do ambiente de trabalho do } \\
\text { enfermeiro em plataforma de petróleo offshore }\end{array}$ \\
\hline & $\begin{array}{l}\text { Trabalho e transtornos mentais: uma análise da } \\
\text { saúde mental dos petroleiros da Bacia de Campos } \\
\text { frente às transformações no mundo do trabalho }\end{array}$ \\
\hline & $\begin{array}{l}\text { Trabalhador portuário: perfil de doenças } \\
\text { ocupacionais diagnosticadas em serviço de saúde } \\
\text { ocupacional }\end{array}$ \\
\hline & $\begin{array}{l}\text { Discutindo e refletindo sobre a competência do } \\
\text { enfermeiro offshore }\end{array}$ \\
\hline & $\begin{array}{l}\text { Trabalho do enfermeiro offshore e a interface da } \\
\text { cultura de segurança }\end{array}$ \\
\hline & $\begin{array}{l}\text { As competências do enfermeiro no processo de } \\
\text { trabalho em plataforma offshore de petróleo }\end{array}$ \\
\hline & $\begin{array}{l}\text { Enfermeiro embarcado em plataforma petrolífera: } \\
\text { um relato de experiência offshore }\end{array}$ \\
\hline
\end{tabular}

Fonte: Próprio Autor

$\mathrm{Na}$ sexta fase, foi demonstrada a revisão e síntese do conhecimento que foi visto com base nas produções analisadas, sendo possível observar, contar e somar, descrever e qualificar os dados, para aglomerar o conhecimento produzido através da temática ${ }^{12}$. 
O conteúdo foi direcionado para atuação do enfermeiro nas ações da promoção, prevenção de doenças ocupacionais e manutenção da saúde dos trabalhadores embarcados, abrangendo o que contribui para os programas e assistência necessária para avaliação das condições do trabalhador durante o período de trabalho, descanso ou lazer na plataforma.

\section{Atuação do enfermeiro nas ações da promoção, prevenção de doenças ocupacionais e manutenção da saúde dos trabalhadores embarcados}

Para simples esclarecimento, Leite $^{8}$ define os riscos ocupacionais sendo "todas as situações de trabalho que podem romper o equilíbrio físico, mental e social das pessoas, e não somente as situações que originem acidentes e doenças".

Cabe ao enfermeiro a inspeção da condição dos equipamentos de proteção indivídual utilizados pelos trabalhadores baseado na NR6 que preconiza que as empresas são obrigadas a fornecer aos empregados, gratuitamente, esses equipamentos adequados ao risco, e em perfeito estado de conservação e funcionamento ${ }^{13}$.

A base de ação do enfermeiro do trabalho é preventivo e promocional, pautada na educação em saúde, permanente, continuada, e treinamento em serviço. Vendruscolo et al. ${ }^{6}$ corroboram dizendo que "as atividades educativas são construídas a partir da análise coletiva dos processos de trabalho, levando em conta as necessidades especificas de profissionais e equipes".

A enfermagem do trabalho é vista como uma profissão, segundo Leite ${ }^{8}$, "destinada a proporcionar cuidados e atenção aos trabalhadores, não importando a função que desempenham. Sua saúde e segurança são fundamentais para que possam desenvolver suas atividades de forma satisfatória no decorrer do dia-a-dia".

O que nos reportamos para a atuação do enfermeiro em desenvolver ações de promoção e proteção à saúde a partir do planejamento em saúde, das situaçõesproblema relacionadas à saúde dos trabalhadores offshore no atendimento em casos de menor para maiores complexidades, tendo como suporte de emergência um leito, equipamentos e medicamentos diversos ${ }^{14}$.

Por isso que o enfermeiro, nas atividades preventivas de agravos e acidentes, estimula a mudança de estilo de vida desses trabalhadores com a finalidade de 


\section{Revisão}

\section{Saúde Funcional}

promover a qualidade de vida, para produzir estratégias direcionadas na orientação de alimentação, inserção de atividade física, controle do estresse aos profissionais, numa linguagem que alcance a todos os trabalhadores de modo que as informações sejam compreendidas no coletivo ${ }^{15}$.

Vendruscolo et al. ${ }^{6}$ revelam que a "troca de saberes entre os sujeitos envolvidos e na aplicabilidade do processo educativo no dia-a-dia do trabalho em saúde. O aprender e o ensinar estão indissociáveis e implicam na inevitável mudança da realidade".

Desta forma, o enfermeiro do trabalho tem uma atuação bastante ampla dentro das organizações. Entre suas funções primordiais estão a liderança da equipe, pois na maioria das vezes temos o técnico de enfermagem que tem suas atribuições voltadas para a assistência, direcionado pelo enfermeiro offshore. Já o enfermeiro do trabalho tem suas orientações voltadas para a promoção da saúde e prevenção de agravos que possam acontecer durante o perído de serviço dos trabalhadores, tais como lesão por esforço repetitivo e distúrbio osteomuscular ${ }^{16}$.

A preservação da saúde do trabalhador é primordial, pelas ações de enfermagem em um trabalho coletivo por meio de divulgação de informações básicas de prevenção, com finalidade de assegurar a saúde na plataforma, articulando os subprocessos de trabalho. Por isso que profissional da saúde procede o atendimento de saúde por demanda espontânea, ao sinalizar um mal-estar ou a ocorrência de alguma emergência ${ }^{17}$. De acordo com Marziale ${ }^{18}$, a intervenção da enfermagem é de:

Grande importância, uma vez que o fundamento na execução de programa preventivo proporcionará ao trabalhador uma satisfação pessoal maior, ao se sentir seguro e protegido. Neste estudo foi desenvolvido um tema atual, buscando esclarecer como as empresas têm utilizado o enfermeiro do trabalho em uma atuação preventiva e promotora da saúde e da qualidade dos ambientes de trabalho, visando proteger a saúde dos trabalhadores. É importante que o offshore reconheça seu papel técnico, político, social e ético e compreenda o processo saúde-doença para desenvolver e executar ações preventivas com o objetivo de melhorar a qualidade de vida.

É muito importante promover atividades que possibilitam o bem-estar para todos os trabalhadores embarcados. A enfermagem do trabalho, como um ramo da saúde pública, tem como prioridade proteger contra os riscos decorrentes de suas atividades laborais; proteger contra agentes químicos físicos, biológicos e 


\section{Revisão}

\section{Saúde Funcional}

psicossociais; a manutenção da saúde no mais alto grau do bem-estar físico e mental e a recuperação e lesões, doenças ocupacionais ou não-ocupacionais reabilitando os indivíduos para o trabalho ${ }^{14}$.

Almeida et al. ${ }^{19}$ reforçam a importância da operacionalização e planejamento "das ações de prevenção e reabilitação construídas pelos profissionais da saúde, tendo em vista a complexidade que envolve a análise e o reconhecimento dos danos à saúde que podem ser suscitados pelos riscos heterogêneos".

O enfermeiro offshore executa atividades na elaboração de estratégias preventivas e melhorias das condições de trabalho, utilizando elementos administrativos e gerenciando todas para integrar ações e alcançar a meta do trabalho em saúde em alto mar, viabilizando a qualidade da saúde, por levar informação, atenção e cuidados a todos, de maneira clara e objetiva, priorizando as ações de prevenção. Uma vez orientados e preparados, os trabalhadores tornam-se multiplicadores ${ }^{20}$.

Os profissionais da saúde embarcados podem sofrer com agravos também, principalmente pela plataforma, que é considerada uma área confinada e passiva de acidentes, onde, de acordo com Almeida et al. ${ }^{19}$, a maioria dos estudos tem seu foco voltado para os "fatores causadores de doenças e à ocorrência de acidentes de trabalho, sobretudo entre os próprios profissionais da área da saúde".

Neste citação podemos perceber a atenção global, não só focando nos trabalhadores offshores, mas envolvendo a equipe de profissionais da saúde embarcados, que estão compartilhando o mesmo ambiente, com todas as exposições.

Mas, ao analisar a atuação do enfermeiro frente às doenças ocupacionais que podem atingir os trabalhadores na embarcação, podem ser relacionados à ocupação, em função da exposição de agentes ou condições desencadeadoras, exigindo padrões mínimos em determinadas funções desempenhadas a oferecer o menor risco possível. Os fatores relacionados às doenças podem ser o ambiente, características físicas e psicológicas, instrumentos de trabalho e os riscos físicos, químicos, ergonômicos, mecânicos, biológicos e psicossociais no cotidiano ${ }^{8}$.

Os agentes físicos mais nocivos à saúde dos trabalhadores são os ruídos, vibrações e temperaturas. Já entre os fatores químicos pode-se citar a poeira, gases, havendo ainda os fatores biológicos, comuns diante dos contatos no dia-a-dia das pessoas, e ainda os ergonômicos, sem ainda deixar de mencionar o estresse 


\section{Revisão}

\section{Saúde Funcional}

ocupacional. Quando os sintomas de estresse começam a aparecer é necessário buscar auxílio médico para tratar os sintomas físicos decorrentes. $O$ estresse tem se tornado em nosso cotidiano um fato comum ${ }^{14}$.

Portanto, atividades durante o expediente de trabalho trazem benefícios para os trabalhadores, para a melhoria dos vícios posturais, promoção do bem-estar geral, combate ao sedentarismo, diminuição, controle e monitorização do estresse ocupacional, como também as Lesões por Esforço Repetitivo / Distúrbio Osteomuscular Relacionado ao Trabalho (LER/DORT) ${ }^{21}$.

Portanto, Leite ${ }^{8}$ diz que "o papel do enfermeiro de trabalho abarca tudo o que rodeia a saúde do trabalhador, desde da forma de actuação do enfermeiro no serviço bem como medida de prevenção de acidentes e doenças no próprio local de trabalho".

Para todas essas ações se faz necessário, de acordo com Almeida et al. ${ }^{19} \mathrm{a}$ obtenção de dados "concretos e integrados a respeito do ambiente e da situação de saúde dos sujeitos em questão, para a produção de conhecimento e planejamento de intervenções em enfermagem aos trabalhadores portuários".

Assim, o enfermeiro do trabalho não trabalha sozinho. Um trabalho em conjunto com o enfermeiro, o técnico de enfermagem e a equipe multidisciplinar offshore são fundamentais na vigilância dos problemas que podem aparecer voltados ao trabalhador.

\section{CONCLUSÃO}

É necessário que o enfermeiro do trabalho seja capaz de avaliar, planejar e executar, através da vigilância em saúde, os riscos presentes no ambiente de trabalho, promovendo o desenvolvimento da educação contínuada para a segurança no trabalho na plataforma, que é marcada pelo processo de reestruturação produtiva e flexibilização das relações de trabalho, que podem intensificar os desgastes dos trabalhadores.

Sendo assim, ele é motivado para o aumento da produtividade, através das ações que colaboram com a coletividade, buscando a qualidade de vida, promoção da saúde, manutenção da integridade fisíca e psicológica, viabilizando ações não apenas preventivas, mas saudáveis para todos os trabalhadores embarcados. 


\section{Revisão}

\section{Saúde Funcional}

Foi possível identificar que o enfermeiro tem um papel importante na prevenção de agravos e proteção contra os riscos de doenças ocupacionais na plataforma, prestando assistência de enfermagem a manutenção da saúde do trabalhador e a qualidade de vida.

Cabe ressaltar que existem poucas produções na Biblioteca Virtual em Saúde e é necessário estimular a produção, ainda mais diante de 17.464 estudos, dos quais apenas foi possível extrair 07 pesquisas, baseado na revisão integrativa, mediante aos descritores: Enfermagem; Qualidade de vida; Saúde do trabalhador; Plataforma, pois não existe os descritores Offshore ou offshores no DeCS - Terminologia em Saúde, dificultando a busca.

\section{REFERÊNCIAS}

1. Amorim GH, Guedes MAS, Guedes CCP, Aguiar BGC. Enfermeiro embarcado em plataforma petrolífera: um relato de experiência offshore. Emferm. 2013;22(1):25765.

2. Martinho MR. Trabalho e transtornos mentais: uma análise da saúde mental dos petroleiros da Bacia de Campos frente as transformações no mundo do trabalho. Escola de Serviço Social: Universidade Federal Fluminense; 2012.

3. Ribeiro MCS. Enfermagem e trabalho: fundamentos para a atenção à saúde dos trabalhadores. Enfermagem e trabalho: fundamentos para a atenção à saúde dos trabalhadores. São Paulo: Martinari; 2008.

4. Delgado LB. A saúde masculina no pronto atendimento e na atenção básica: intervenção no município de Navegantes/SC [Dissertação]. Programa de Mestrado Profissional em Saúde e Gestão de Trabalho: Universidade do Vale do Itajaí; 2013.

5. Chagas AMR, Salim CA, Santos LM. Saúde e segurança no trabalho no Brasil: aspectos institucionais, sistemas de informação e indicadores. In: Aplicada IdPE, editor. Ipea: Ministério do Trabalho e Empregos; 2011.

6. Vendruscolo C, Anastácio CA, Zocche DAA, Trindade LL, Kloh D. Concepção de coordenadores da atenção básica sobre educação permanente em saúde: aproximações e distanciamentos com pressupostos freireanos. Inova Saúde. 2015;4(1):47-69.

7. Ferreira JC, Silva Júnior A, Assis ZV. Saúde a bordo. Rev Emergência. 2010;12:31-41.

8. Leite R. Riscos ocupacionais para os profissionais de enfermagem. Portaldoconhecimento.org: Escola Superior da Saúde Mindelo; 2014. 
9. Chen WQ, Yu IT, Wong TW. Impact of occupational stress and other psychosocial factors on musculoskeletal pain among Chinese offshore oil installation workers. Occup Environ Med. 2005;62(4):251-6.

10. Vilela PF, Souza AC. Liderança: um desafio para o enfermeiro recém-formado. Rev enferm UERJ. 2010;18(4):591-7.

11. Minayo SMC. O desafio do conhecimento. Pesquisa qualitativa em saúde. Abrasco. 1993(2):269.

12. Souza MT, Silva MD, Carvalho R. Revisão integrativa: o que é e como fazer. Einstein (São Paulo). 2010;8(1):102-6.

13. Brasil. Norma Regulamentadora N6. Portaria GM n. 3.214 , de 08 de junho de 1978. Dispõe sobre o uso de Equipamento de Proteção Individual. In: Emprego MT, editor. Portaria SIT n. 25, de 15 de outubro de 2001: Segurança e Saúde no Trabalho.; 1978.

14. Guedes MAS, Guedes CCP, Pittioni RB. Trabalho do enfermeiro offshore e a interface da cultura de segurança. Rev Acred. 2012;2(4):60-76.

15. Soares M. Ergonomia: soluções e propostas para um trabalho melhor. Produc. $2009 ; 19(3)$.

16. Castro MR, Farias SNP. A produção científica sobre riscos ocupacionais a que estão expostos os trabalhadores de enfermagem. Esc Anna Nery Rev Enferm. 2008;12:364-9.

17. Guedes CCP, Aguiar BGC. Discutindo e refletindo sobre a competência do enfermeiro offshore. Rev enferm UERJ. 2012;20(1):61-6.

18. Marziale MHP. Contribuições do enfermeiro do trabalho na promoção da saúde do trabalhador. Acta paul enferm. 2010;23(2).

19. Almeida MCV, Cezar-Vaz MR, Rocha LP, Cardoso LS. Trabalhador portuário: perfil de doenças ocupacionais diagnosticadas em serviço de saúde ocupacional. Acta paul enferm. 2012;25(2):270-6.

20. Pereira CC, Costa BG. Empregabilidade do enfermeiro offshore na indústria. Rev paran dig. 2011(14).

21. Abreu SC, Silva MR, Gorgatti ECAS. Ginástica laboral como ferramenta dos programas de qualidade de vida no trabalho (PQVT). S a r e. 2009;4(4):113-32. 